\title{
Electromagnetic shielding performance of CSA- PANI/PAN electrospun fiber membrane added with CB and CNT
}

\begin{abstract}
Electromagnetic radiation protective clothing is the main means of individual protection of electromagnetic radiation, and the ESPESF plays a key role. In this paper, $8 \%, 10 \%$, and $12 \%$ pure PAN electrospinning fiber membranes were prepared, and $0.5 \%$ and $1 \%$ CSA were added to a pure PAN solution in DMF. The fibrous membrane was used to determine the most stable electrospinning process. Subsequently, $1 \%$, $2 \%, 3 \%, 4 \%$, and $5 \%$ carbon black $(\mathrm{CB})$ was added to the $10 \%$ PAN and $1 \%$ CSAPANI solution to prepare electrospinning. Fibrous film, and later added $0.5 \%, 1 \%$, $1.5 \%, 2 \%, 2.5 \%$ of carboxy Carbon nanotube and hydroxyl Carbon nanotube in a $10 \%$ PAN and $1 \%$ CSA-PANI solution to prepare composite nanofiber membranes, Finally, the effect of the addition of various carbon materials on the electromagnetic shielding performance of electrospun fiber membranes was comparatively studied. The shielding properties of polyaniline fiber membranes added with Carbon nanotube were improved. At the same concentration, the electromagnetic shielding performance of the CNT (COOH-)/CSA-PANI/PAN fiber membrane was similar to that of the C/ CSA-PANI/PAN fiber membrane. The electromagnetic shielding performance of the CNT $(\mathrm{OH}-) / \mathrm{CSA}-\mathrm{PANI} / \mathrm{PAN}$ fiber membrane is much better than that of the C/CSAPANI/PAN fiber membrane.
\end{abstract}

Keywords: electromagnetic shielding performance, CSA doped PANI, CSA doped PANI, carbon nano tube
Volume 4 Issue 4 - 2018

\author{
Taohai Yan, 1,2,3 Yajing Shi, 2,3 Dongsheng \\ Chen, ${ }^{2,3}$ Jinhua Jiang,' Nanliang Chen' \\ 'College of Textiles, Donghua University, China \\ ${ }^{2}$ Faculty of Clothing and Design, Minjiang University, China \\ ${ }^{3}$ Fujian Key Laboratory of Novel Functional Fibers and Materials, \\ Minjiang University, China
}

Correspondence: Nanliang Chen, College of Textiles, Donghua University, Shanghai 201620, P.R. China, Tel 021-67792264, Email nlch@dhu.edu.cn

Received: June 14, 2018| Published: July 10, 2018

\section{Introduction}

In 1862, polyaniline was first used as a dyestuff in H.Letheby's research. The research of poliyaniline in the following century had gone through 3 phases: The discuss on "Aniline Black" as a dyestuff, the research of organic semiconductor and the extensive studies of conducting polymer. Due to the lack of profound theoretical knowledge of macromolecular at that time, the further study of polyaniline had been dragged on for nealy a century. Until 1976, MacDiarmid and his stuff from University of Pennsylvania restudied polyaniline and found some unique properties as electric conduction, therefore polyaniline was considered as a conductive polymer which would be most likely widespread in real life. Polyaniline has many excellent characters including easy obtainment of the raw material, easily manipulated synthetic process, and high conductivity, and unique doping performance, excellent electromagnetic microwave absorbing performance, environmental stability, chemical stability and optical properties. Nowadays the synthetic material on the basis of the polyaniline was widely used in such high-tech sectors like marine antifouling, all plastic metal anticorrosion technology, electromagnetic shielding, stealth technology, antistatic technology and sensor components, electroluminescent, solar battery, secondary battery materials, catalytic materials and anti-corrosion materials.

As a polymer that is inherently conductive, it has a high degree of stringent skeletal characteristics of aromatic molecules and is present in a relatively short molecular weight form, so that the viscosity of the solution is generally difficult to directly use electrospinning to prepare polyaniline fibers. ${ }^{1}$ However, polyaniline is the most studied conductive polymer because of its relatively easy synthesis, high conductivity, protonization, and relative environmental stability. ${ }^{2}$
Factors that affect the conductivity of PANI are temperature, ${ }^{3}$ solvents, dopants, humidity, ${ }^{4,5}$ structural defects, ${ }^{6}$ polymer morphology, ${ }^{7}$ But the biggest influence factor is the proton doping level. ${ }^{8}$ The maximum conductivity of PANI is that when it is $50 \%$ doped with protons, the partial amines are protonated above $50 \%$, and when less than $50 \%$, some imines have not been protonated. In both cases, the delocalized charge carriers on the polymer backbone are destroyed, resulting in a decrease in the overall conductivity of the polymer. ${ }^{9}$ In order to avoid the characteristics of difficult spinning of polyaniline electrospinning, in order to improve its conductivity, and expand its application fields, researchers have made a lot of efforts.

Carbon nanotube (CNT) was electrospinned directly without surface functionalization, and they are easily dispersed unevenly. Spinning effects may be poor, and beaded fibers are easily formed. The MWNT and the polyurethane were added to N,N-dimethylformamide (DMF), and the fiber membrane was prepared by electrospinning. The irregularity of the fiber thickness of the fiber membrane was very high. ${ }^{10}$ Many literatures confirm that the surface functionalization of CNT facilitates electrospinning, it was confirmed that MWNT$\mathrm{COOH}$ was formed after oxidative acidification of MWNTs, the entanglements between the tubes weakened, and the dispersion was enhanced, ${ }^{11}$ After the multi-walled Carbon nanotube were first treated with nitric acid and then treated with hydrochloric acid, the surface of the multi-walled Carbon nanotube was carboxylated, and the prepared carbon nanotube-adsorbed nylon nanofiber membrane had a conductivity of $0.15 \mathrm{~S} / \mathrm{cm},{ }^{12}$ The surface of MWNTs can be treated with sulfuric acid and nitric acid first, then treated with thionyl chloride $\left(\mathrm{SOCl}_{2}\right)$ solution, and finally treated with ethylenediamine solution to achieve amino functionalization of MWNTs. ${ }^{13}$ 
Researchers pay more attention to the effects of carbon-based materials on the electrical conductivity of fibers, conduct qualitative and quantitative studies on them, explore their impact mechanisms, and seek basic theoretical support. The researchers found that adding only a small amount of Carbon nanotube significantly improves the conductivity; Kim YT used electrospinning technology to prepare SWCN/PVDF nanofiber membranes. ${ }^{14}$ It was found that the critical point of insulator-to-conductor transition was $0.04 \mathrm{wt} \%$ SWCNT content. ${ }^{15}$ Study confirms that the electrical conductivity of ordered electrospun PMMA nanofibers with $2 \%$ added MWCNTs is 10 orders of magnitude higher than pure PMMA fiber. ${ }^{16}$ The effect of the content of SWCNT, DWCNT and MWCNTs on the performance of electrospun nanofibers was studied. The trifluoroacetic acid (TFA) and dichloromethane (DCM) solutions were dissolved in PET, and the Carbon nanotube was uniformly dispersed in the solution. Electrospinning was used to prepare PET-CNT nanofibers. It was found that the mechanical properties of SWCNT with the same mass fraction were better than MWCNTs, but the conductivity of SWCNT with the same mass fraction was worse than that of MWCNTs. ${ }^{17,18}$

Electrospinning is a process that uses electrostatic forces to form fibers. ${ }^{19}$ This process uses a high voltage supply, which induces the charge of a certain polarity into a polymer solution or a polymer melt that is accelerated to a collector that has the opposite polarity. The obtained electrospun fibers have a small diameter; in the micrometer to nanometer range. ${ }^{20}$ The high surface area per unit mass and high porosity of electrospun fibers are advantageous for applications in many fields. In fact, the electrospun fibers have potential uses in various applications such as filtration and protective clothing, as well as biomedical and tissue engineering scaffolds that includes drug delivery systems..$^{21,22}$

In this paper, $8 \%, 10 \%, 12 \%$ concentration of pure PAN electrospinning fiber membranes were prepared, and $0.5 \%$ and $1 \%$ CSA doped polyaniline was added in $10 \%$ PAN solution for electrospinning fiber membranes, and then added $1 \%, 2 \%, 3 \%, 4 \%$, $5 \% \mathrm{CB}$ in $10 \%$ PAN and $1 \%$ CSA-PANI solution, and later added $0.5 \%, 1 \%, 1.5 \%, 2 \%$ and $2.5 \%$ of carboxyl Carbon nanotube and hydroxyl Carbon nanotube were added in 10\% PAN and 1\% CSAPANI solution for electrospinning, and studied the electromagnetic shielding performance of all of the electrospun fiber membranes.

\section{Materials and methods}

\section{Materials and equipment}

Camphorsulfonic acid (CSA) and polyacrylonitrile (PAN) (MW 150,000 ) was purchased from TCI (Shanghai, China). CNT was purchased from Beijing Boyu High-tech Materials Technology Co., Ltd. (Beijing, China). Carbon black (CB) was purchased from Damao Chemical Reagent Factory (Tianjin, China). N,N-Dimethylformamide (DMF) was purchased from Tianjin Zhiyuan Chemical Reagent Co., Ltd (Tianjin, China). All chemicals were analytical reagent and were used without alteration. Ultrasonic cleaner CD-4800 was purchased from Changzhou Ruipin Precision Instrument Co., Ltd.. (Changzhou, China). The high pure water system EXPLORER was purchased from Chenhuang Scientific Instrument co., LTD (Shanghai, China). The high voltage DC power supply DW-P503-1ACDF was purchased from Dongwen High Voltage Power Supply co., LTD (Tianjin, China). The two-channel trace injection pump WZS-50F6 was purchased from Smith Medical Instrument co., LTD (Zhejiang, China).

\section{Methods}

Separately weigh $8 \%, 10 \%$, and $12 \%$ of the PAN powder in a reagent bottle containing an appropriate amount of DMF. After the PAN powder was dissolved in a magnetic stirrer at a constant temperature of 50 degrees, it was ultrasonically dispersed for 30 minutes, magnetic stirring was continued for 12 hours, and the fully dissolved PAN solution was deeper than the previous yellowish one, and the electrospun membrane was prepared using a self-made electrospinning apparatus. Take appropriate mass fractions of PAN, $8 \%, 10 \%$, and $12 \%$, and weigh equimolar polyaniline and camphor sulfonic acid (CSA) into PAN solution. The content of doped polyaniline is $0.5 \%$ and $1 \%$. The mixed solution was stirred on a magnetic stirrer for 12 hours, and an electrospun membrane was prepared using a self-made electrospinning apparatus. Taked appropriate amount of $10 \%$ PAN and $1 \%$ doped polyaniline solution, added CB $1 \%, 2 \%, 3 \%, 4 \%, 5 \%$, $6 \%$ and $7 \%$ respectively, added CNT (COOH-) and CNT (OH-) $0.5 \%$, $1 \%, 1.5 \%, 2 \%$ and $2.5 \%$ respectively. The mixed solution was stirred for 12 hours and an electrospun membrane was prepared using a selfmade electrospinning apparatus.

\section{Electromagnetic shielding test}

Electromagnetic shielding performance testing instrument uses DR-913G fabric anti-electromagnetic radiation performance tester, each sample size is $18 \mathrm{~cm} \times 18 \mathrm{~cm}$ square sample, electromagnetic shielding data was got from the DR-913G fabric anti-electromagnetic radiation performance tester.

\section{Results and discussion}

The value of fabric electromagnetic shielding shows that the smaller the negative amplitude of fiber radiation, the greater the absolute value and the smaller the amount of transmission, ie, the better the electromagnetic shielding performance. According to the formula.

$$
\mathrm{S}_{e}=-10 \log \left(\mathrm{S}_{B} \div \mathrm{S}_{A}\right)
$$

In the formula: $\mathrm{S}_{e}-$ Electromagnetic shielding amplitude $(\mathrm{dB})$

$$
\mathrm{S}_{B}-\text { Transmission }
$$

$\mathrm{S}_{A}-$ Reflection amount

It is relatively straightforward to represent the trend of shielding performance with the line graph of absolute value of the absolute value. The line chart intuitively shows the tendency of electromagnetic shielding performance with different concentration of fiber membrane radiation amplitude at three different frequencies of $1 \mathrm{MHz}, 30 \mathrm{MHz}$ and $300 \mathrm{MHz}$. Figure 1 represents an electrospun fibrous membrane prepared by the electromagnetic shielding curve of the control group at a PAN concentration of $10 \%$ and a CSA doped polyaniline concentration of $1 \%$. When the frequency is $1 \mathrm{MHz}$, the radiation amplitude is $-30 \mathrm{~dB}$, and when the frequency is $30 \mathrm{MHz}$, the radiation amplitude is $-20.4 \mathrm{~dB}$, and when the frequency is $300 \mathrm{MHz}$, the radiation amplitude is $-3.4 \mathrm{~dB}$. The change in the $\mathrm{CB}$ concentration at $30 \mathrm{MHz}$ and $300 \mathrm{MHz}$ in Figure 2 shows a relatively gradual change in the electromagnetic shielding performance. At $1 \mathrm{MHz}$, the difference in the variation can be seen and the radiation amplitudes are $-31,-39,-57,-158,-26,-1 \mathrm{~dB}$, respectively. The electromagnetic shielding performance of the polyaniline composite fiber with $\mathrm{CB}$ was improved. Among them, when the $\mathrm{CB}$ concentration was $3 \%$ and 
$4 \%$, the transmission amount was very small, and the electromagnetic shielding performance was relatively best. It can be seen from the Figure 2 that the electromagnetic shielding performance of the fiber membrane with different radiation frequencies is approximately the same with the concentration change trend, and the trend changes significantly at a frequency of $1 \mathrm{MHz}$.

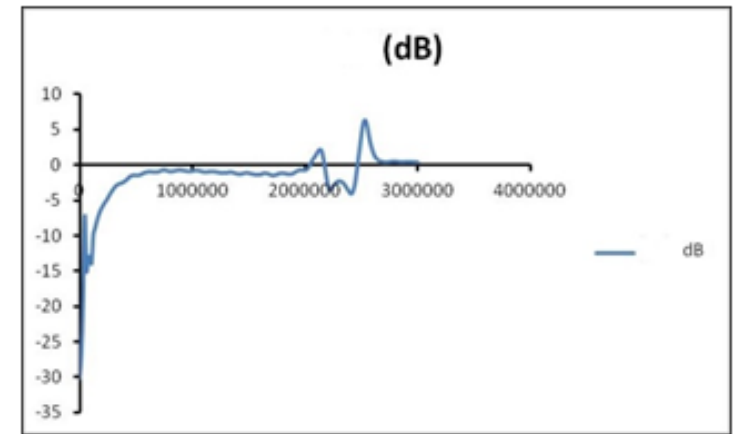

Figure I Electromagnetic shielding curve of $10 \%$ PAN and I\% CSA-PAN electrospun fiber membrane.

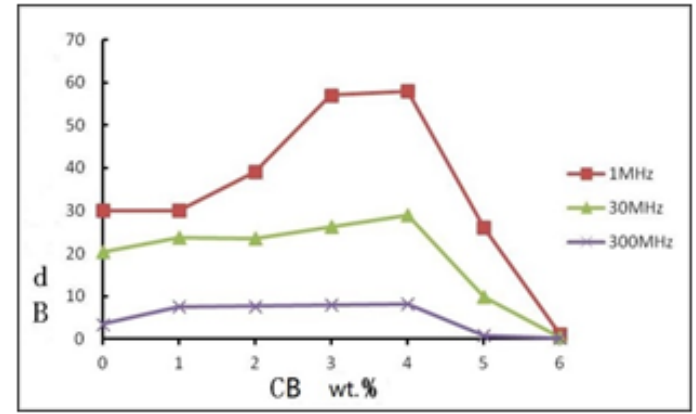

Figure 2 The relationship between CB concentration and electromagnetic shielding performance.

Figure 3 shows the electromagnetic shielding performance at a frequency of $30 \mathrm{MHz}$ and $300 \mathrm{MHz}$ when the carboxyl carbon nanotube concentration is changed, and the change in the curve tendency is gentle. At $1 \mathrm{MHz}$, the trend of electromagnetic shielding performance changes significantly, with radiation amplitudes of -56 , $-59,-53,-37$, and $-31 \mathrm{~dB}$ respectively. The shielding performance of polyaniline composite fiber membranes with carboxyl Carbon nanotube was improved compared with the $10 \%$ PAN and $1 \%$ CSAPANI. The concentration of carboxyl Carbon nanotube increased, and the electromagnetic shielding performance of fibers showed a decreasing trend.

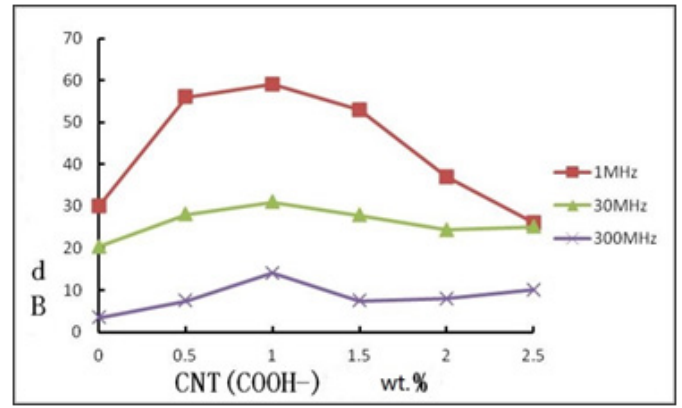

Figure 3 The relationship between the concentration of CNT (COOH-) and electromagnetic shielding performance.
Figure 4 shows the EMI shielding effectiveness of $\mathrm{CNT}(\mathrm{OH}-)$ at three different frequencies. The performance trends are approximately the same. At $1 \mathrm{MHz}$, the radiating amplitudes are $-37,-38,-42,-62$, and $-40 \mathrm{~dB}$, respectively. The shielding performance of the polyaniline composite fiber membrane added with hydroxyl Carbon nanotube was improved compared with the control group. With the increase of the concentration of hydroxyl Carbon nanotube, the electromagnetic shielding performance of fibers is generally on the rise. The electromagnetic shielding performance is best when the concentration of Carbon nanotube is $2 \%$.

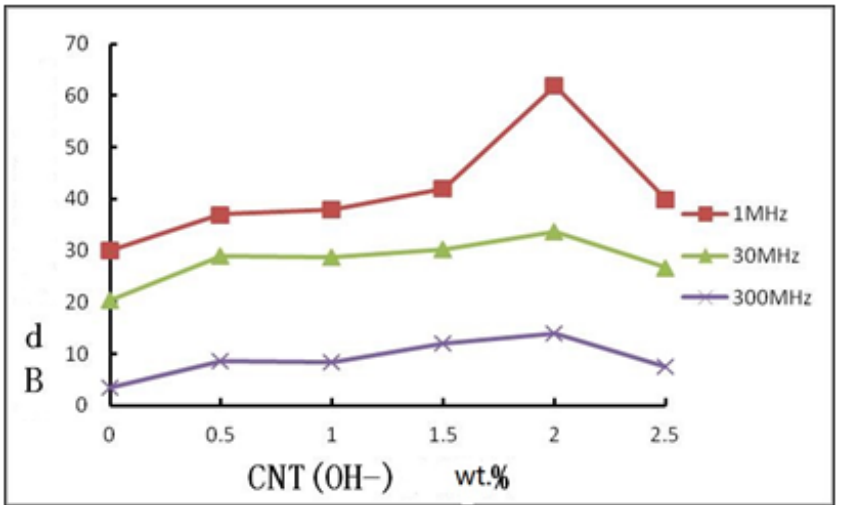

Figure 4 The relationship between the concentration of CNT (OH-) and electromagnetic shielding performance.

Figures 5-7 represents the electromagnetic shielding performance of C/CSA-PANI/PAN and CNT/PANi/PAN fiber membranes at different frequencies. At the same concentration, the electromagnetic shielding performance of C/CSA-PANI/PAN fiber membrane is better than that of CNT/CSA-PANI/PAN fiber membrane. The electromagnetic shielding performance of the $\mathrm{CNT}$ (COOH-)/ CSA-PANI/PAN fiber membrane and the electromagnetic shielding performance of the C/CSA-PANI/PAN fiber membrane are comparable, but the electromagnetic shielding ratio of the $\mathrm{CNT}(\mathrm{OH}-) / \mathrm{CSA}-\mathrm{PANI} /$ PAN fiber membrane is C/CSA-PANI/PAN fiber membrane has much better electromagnetic shielding performance in.

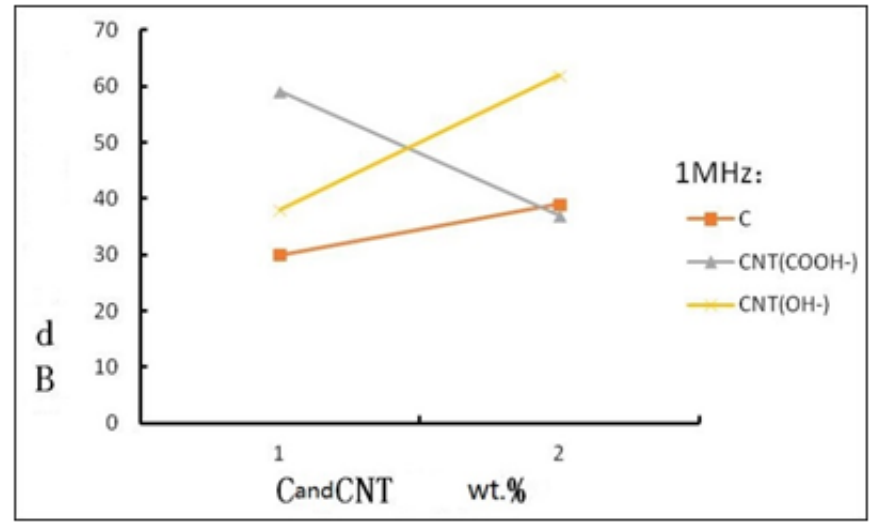

Figure 5 electromagnetic shielding performance of C/CSA-PANI/PAN and CNT/CSA-PANI/PAN fiber membranes at IMHz. 


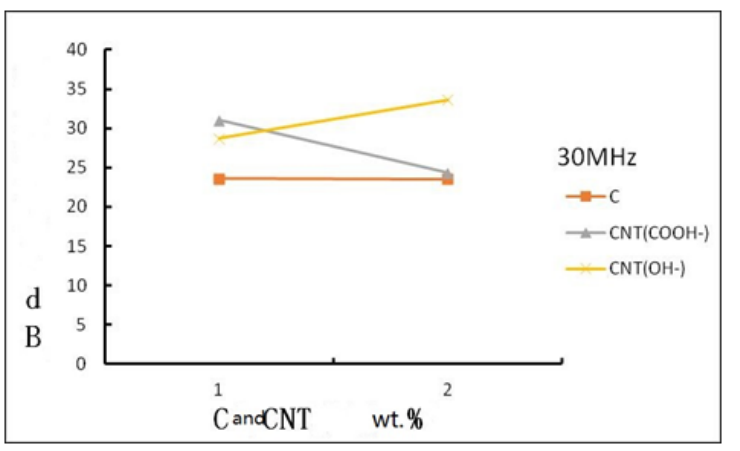

Figure 6 electromagnetic shielding performance of C/CSA-PANI/PAN and CNT/CSA-PANI/PAN fiber membranes at $30 \mathrm{MHz}$.

\section{Conclusion}

Under the same external conditions, when the concentration of PAN and doped polyaniline is the same, the spinning solution containing $\mathrm{CB}$ is more viscous than the spinning solution containing Carbon nanotube, and the $\mathrm{CB}$ concentration increases, and the spinning solution will follow. Become viscous. The viscosity of the solution can affect the electrospinning efficiency. C/CSA-PANI/PAN spinning films are brittle compared to CNT/CSA-PANI/PAN and are brittle. Compared with the electromagnetic shielding performance of the 10\% PAN and 1\% CSA-PANI fiber membrane in the control group, the shielding performance of the polyaniline composite fiber with $\mathrm{CB}$ was improved, and the transmission was very low when the $\mathrm{CB}$ concentration was $3 \%$ and $4 \%$. Electromagnetic radiation performance is relatively best. The shielding properties of polyaniline fiber membranes added with Carbon nanotube were improved. At the same concentration, the electromagnetic shielding performance of the CNT (COOH-)/CSA-PANI/PAN fiber membrane was similar to that of the C/CSA-PANI/PAN fiber membrane. The electromagnetic shielding performance of the CNT (OH-)/CSA-PANI/PAN fiber membrane is much better than that of the C/CSA-PANI/PAN fiber membrane.

\section{Acknowledgements}

None.

\section{Conflict of interest}

Author declares there is no conflict of interest in publishing the article.

\section{References}

1. Yu JH, Fridrikh SV, Rutledge GC. The role of elasticity in the formation of electrospun fibers. Polymer. 2006;47(13):4789-97.

2. Martins CR, de Freitas PS, De Paoli MA. Physical and conductive properties of the blend of polyaniline/dodecylbenzenesulphonic acid with PSS. Polymer Bulletin. 2003;49(5):379-86.

3. Javadi HHS, Cromack KR, MacDiarmid AG, et al. Microwave transport in the emeraldine form of polyaniline. Physical Review B. 1989;39(6):3579.

4. Angelopoulos M, Ray A, Macdiarmid AG, et al. Polyaniline: processability from aqueous solutions and effect of water vapor on conductivity. Synthetic metals. 1987;21(1):21-30.

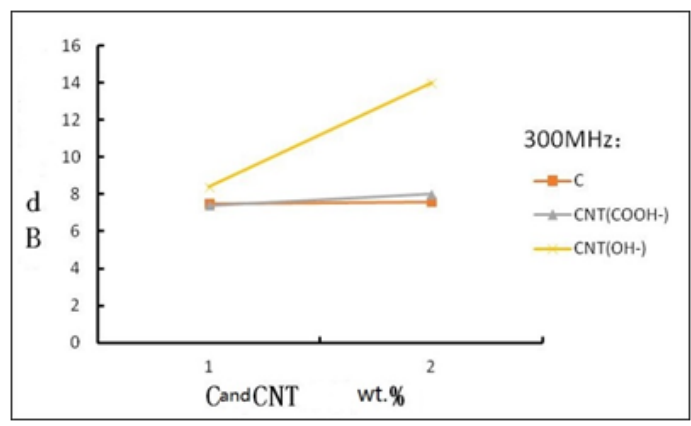

Figure 7 electromagnetic shielding performances of C/CSA-PANI/PAN and CNT/CSA-PANI/PAN fiber membranes at $300 \mathrm{MHz}$.

5. Matveeva ES. Residual water as a factor influencing the electrical properties of polyaniline. The role of hydrogen bonding of the polymer with solvent molecules in the formation of a conductive polymeric network. Synthetic Metals. 1996;79(2):127-39.

6. Thyssen A, Borgerding A, W Schultze J. Formation and electronic conductivity of polyaniline. Makromolekulare Chemie. Macromolecular Symposia. 1987;8(1):143-57.

7. Lu Y, Li J, Wu W. Morphological investigation of polyaniline. Synthetic metals. 1989;30(1):87-95.

8. Chiang JC, MacDiarmid AG. Polyaniline: protonic acid doping of the emeraldine form to the metallic regime. Synthetic Metals. 1986;13(1):193205.

9. Shin MK, Kim YJ, Kim SI, et al. Enhanced conductivity of aligned PANi/ PEO/MWNT nanofibers by electrospinning. Sensors and Actuators B: Chemical. 2008;134(1):122-26.

10. Li Minxuan, Tan Xiaohong. Properties of electrospun composite fibers of polyurethane(PU)/single Wall Carbon Nanotube. J Donghua University: Natural Science Edition. 2013;39(6):703-9.

11. Yin Chuan. Fabrication of conductive PVA/PEDOT:PSS/DMSO Nanofibers by Electrospinning. Huazhong University of Science and Technology; 2013.

12. Kim HS, Jin HJ, Myung SJ, et al. Carbon Nanotube-Adsorbed Electrospun Nanofibrous Membranes of Nylon 6. Macromolecular rapid communications. 2006;27(2):146-51.

13. Zhou Xin. Preparation of electrospun zno-carbon nanofiber composite modified electrodes and its sensing application. Donghua University; 2014.

14. Jeong JS, Jeon SY, Lee TY, et al. Fabrication of MWNTs/nylon conductive composite nanofibers by electrospinning. Diamond and related materials. 2006;15(11):1839-43.

15. Kim YT, Baek CK. Electrospinning of poly (vinylidene fluoride) dimethylformamide solutions with Carbon nanotube. J Polymer Science Part B: Polymer Physics. 2003;41(13):1572-7.

16. Sundaray B, Subramanian V, Natarajan TS, et al. Electrical conductivity of a single electrospun fiber of poly (methyl methacrylate) and multiwalled carbon nanotube nanocomposite. Applied Physics Letters. 2006;88(14):143114.

17. Mazinani S, Ajji A, Dubois C. Morphology, structure and properties of conductive PS/CNT nanocomposite electrospun mat. Polymer. 2009;50(14):3329-42. 
18. Mazinani S, Ajji A, Dubois C. Fundamental study of crystallization, orientation, and electrical conductivity of electrospun PET/carbon nanotube nanofibers. J Polymer Science Part B: Polymer Physics. 2010;48(19):2052-64.

19. Malafeev KV, Moskalyuk OA, Yudin VE, et al. Production of Monofilaments from Polylactide Melt: Structure, Properties, and Biocompatibility. Fibre Chemistry. 2017;48(6): 456-61.

20. Kovalenko GM, Bokova ES, Yu I, et al. Electrospun Fibrous Materials Made of Collagen and Chitin Derivatives. Fibre Chemistry. 2017;48(6):466-69.
21. Malakhov SN, Yu A, Khomenko SI, et al. Method of manufacturing nonwovens by electrospinning from polymer melts. Fibre chemistry. 2009;41(6):355-59.

22. Sidorina AI, Druzhinina TV. Composition and properties of polyacrylonitrile solutions for nanofibre electrospinning processes. Fibre Chemistry. 2012;4494):221-26. 